\title{
ROLE OF OCCUPATIONAL THERAPY IN RESTORING FINE MOTOR SKILLS IN POST STROKE PATIENTS - AN ANALYTICAL REVIEW
}

\author{
Dr. Manish Bhardwaj ${ }^{1}$ \\ $\mathrm{Ph}$. D. Scholar \\ Mahatma Gandhi Occupational Therapy \\ College, Jaipur \\ Address: 49-A, Ganesh Nagar, New Sanganer \\ Road, \\ Sodala, Jaipur-302019
}

\author{
Dr. R. K. Sureka ${ }^{2}$ \\ Prof. \& Head, Department of Neurology, \\ Mahatma Gandhi Medical College \& Hospital \\ Jaipur
}

\author{
Dr. Neha Jain ${ }^{4}$ \\ Assot. Professor \\ Mahatma Gandhi Occupational Therapy \\ College \\ Jaipur
}

Article DOI: https://doi.org/10.36713/epra6138

\begin{abstract}
The occupational therapy has a decisive role in restoring fine motor skills in post stroke patients. The purpose of this study was to compile all the information pertaining to the assessment and treatment performed by occupational therapist. This study amass the complete personification of occupational therapy from the need of theoretical basis to the practical treatment aspects. The study covers the basic idea of stroke and complications arose in the normal movements and leads towards the complete functional aspects of the motor function of human hand. The position of occupational therapy in a rehabilitation team and a detailed knowledge of areas of occupational therapy related to fine motor skills helps to grab the evaluation process used and treatment protocol prescribed to restore fine motor skills after stroke. It helps to gain a detailed know-how of tools and instruments used for assessment and techniques used in the treatment of stroke that focuses on fine motor skills. This study reveals that occupational therapy as its specialty in hand rehabilitation has a wide range of assessments, treatment and management protocols to pursue the treatment of a variety of areas of hand rehabilitation including fine motor skills after an event of stroke.
\end{abstract}

KEYWORDS: fine motor skills, occupational therapy, stroke, hand functions.

\section{INTRODUCTION}

Stroke is the third biggest cause of disability and second biggest cause of death in the world. [Global Health Estimates. Geneva: World Health Organization; 2012]. Stroke is a condition occurring due to sudden death of certain cells of brain due to lack of oxygen when the blood flow is lost or hindered due to blockage or rupture of the arteries of the brain. This condition is called Cerebro Vascular Accident (CVA); i.e. a condition when there occurs a lack of blood supply leading to scarcity of oxygen ultimately causing the death of certain cells of the brain.

Fine motor skills is the ability to control movements of fingers and thumb through specialised movements through the coordinated function of nervous system and small muscles of hand. Alterations in fine motor skills can be caused due to loss of muscle strength, loss of muscle co-ordination, sensory alterations, cognitive disabilities, etc. leading to tremors uncontrolled movements of the wrist, fingers and the thumb thus patients are not able to 
perform the required activities as expected and to the maximum level of functionality. Fine motor skills deficits due to stroke drastically affect the overall quality of life by limiting patients' performance in daily activities, their interaction in society and ability to return to profession.

Occupational Therapy is an art and science that works with a holistic approach with the patients with some disability or health issues that limit them to perform their role in Activities of Daily Living (ADL), family, society, job or business and recreational issues and help them to attain the maximum level of independence.

\section{SIGNIFICANCE OF THE STUDY}

This study focus on the providing a comprehensive knowledge about the working and specialized skillful treatment process in occupational therapy for restoration of fine motor skills in the patients recovering from stroke. This study will help all from a layman to a technical person including occupational therapist as it covers all the theoretical and practical aspects of the treatment of fine motor skills in occupational therapy.

\section{BACKGROUND}

\section{Cause of Stroke}

On the basis of causes of stroke there are two types of stroke:

Ischemic stroke - caused due to lack of oxygen to the cells of the brain. This kind of condition may be a result of narrowing or blockage of blood vessels severely reducing the blood flow to certain parts of the brain.
Hemorrhagic stroke - caused due to rupture or leakage of the arteries of the brain. This condition is generally a result of traumatic brain injuries, high blood pressure, bulges at weak areas in the blood vessel walls (aneurysm), over doses of blood thinners (anticoagulants), deposition of proteins on blood vessel walls leading to their weakening (cerebral amyloid angiopathy).

Stroke leads to many physical, mental and psychological effects like loss of muscle movements (paralysis), speech disorders including dysphagia, cognitive disabilities and memory dysfunctions, psychological stress and emotional discomfort, behavior alterations and loss of self-care abilities. Out of the above listed complications the one which is the most disastrous which makes the patient dependent on others is loss of fine motor skills.

\section{Hand Functions}

Hand Functions are particularly those actions which lead to successful performance of different activities needed to achieve daily routine functions may be single handed or with both hands together. Hand functions are done to achieve four basic actions viz. reach, grip, manipulation/shift and release.

Reach: Action of moving the different joints of upper limb to move the hand towards a certain object to pick or grab it. This action may involve the movement of all or some of the joints and respective muscles.

Grip: The action of holding an object in different positions in hand is referred to as grip. It includes different type grasps and grips.

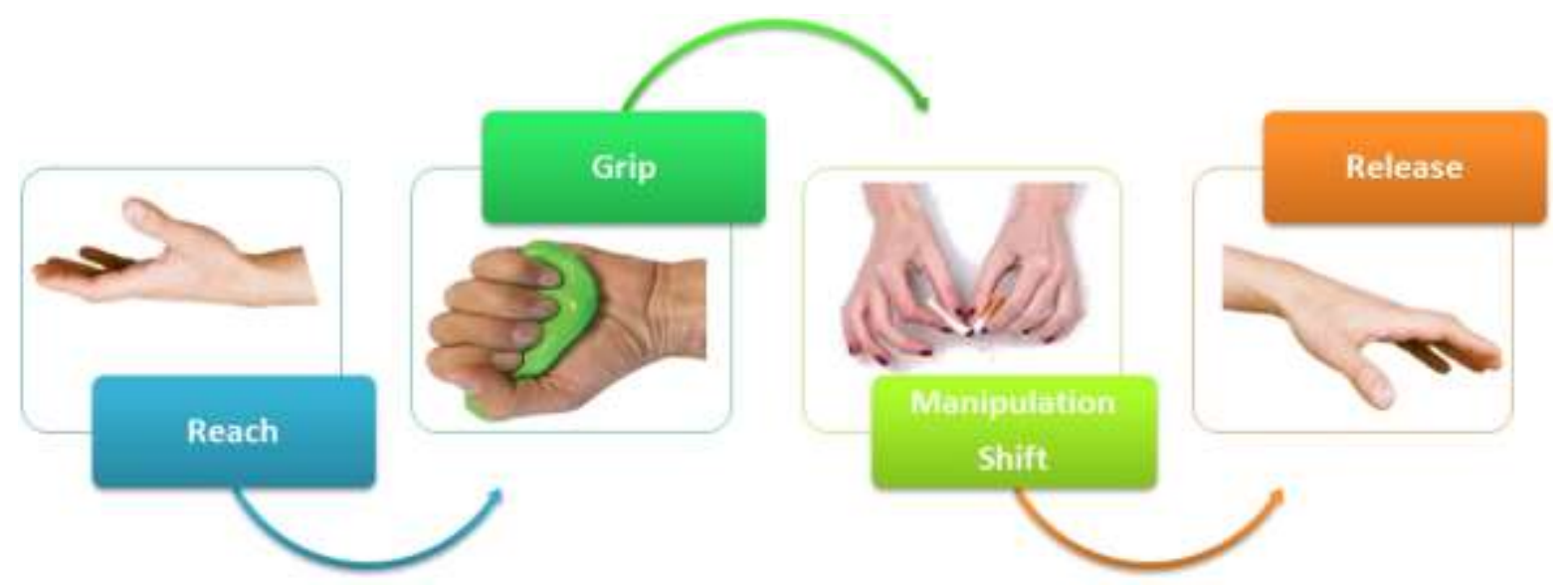

Manipulation/Shift: Placement or change of position of an object in some other position or shapes and sizes in the hand is called manipulation. Its needs specialised movements of fingers and thumb and a good cognitive level for the manipulation of an object in hand. Shift is referred to the change of place of the object in hand from one place to another.
Release: Leaving of an object already in the hand to a required place is called release. This action is done by extending all the fingers and thumb to either full extension or only to the degrees required.

The actions needed for the hand functions are divided into two main categories namely Grasp and Grip and further divided as spherical grasp, cylindrical grasp, hook grasp, lumbrical grasp 
[Lippert, $\mathrm{p}$ 192] and opponance grip, pincer/tip to tip grip and lateral grip.

Grasp

Action of holding an object into the whole hand with the help of most of the parts of hand including fingers, thumb and palm. Grasp is mainly of four types:

Spherical Grasp: It involves the action of all the fingers and thumb in such a way that they make a spherical enclosure that can hold spherical objects like ball, compact disc or a big round lid of a container.

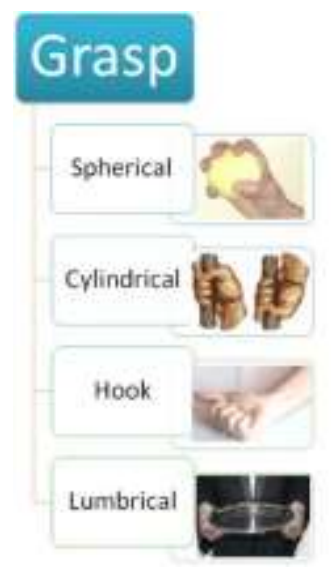

Grip

Action of holding an object with the help of fingers and thumb instead of the whole hand is referred to grip. Grasp is mainly of four types:

Opponance Grip: This is also known as pad to pad grip as the finger pads are used to hold the object. Generally index finger and sometimes the middle finger is used to make an opponance grip along with the pad of thumb.

Pincer Grip: This is also known as tip to tip grip as the tips of the fingers are used to hold the object. The index finger tip and the thumb tip come closer to each other and hold the object. This type of grip is used generally to pick up small objects like pin, marbles, peas, etc.

Lateral Grip: This is a grip can be used to hold a key, a paper or to count currency notes. The thumb pad comes in contact with the lateral aspect of the distal phalanx of the index finger.

Tripod Grip: This is a specialised grip formed by touching the thumb tip to the index finger tip and combined touching with the lateral aspect of the tip of the middle finger. The objects are held in between the apparatus made by this combination. This grip is
Cylindrical Grasp: This grip helps to hold cylindrical objects like pipes, tree branches, etc. All the fingers and the thumb flex to full to hold the object.

Hook Grasp: This grasp looks similar to cylindrical grasp with a free thumb an helps to hold wires and thin cylindrical objects like bucket handles, etc. This grasp needs more power than the cylindrical grasp.

Lumbrical Grasp: Lumbrical grasp is generally used for holding plates and trays in both the hands simultaneously thus it is also called as plate grasp. The fingers are slightly flexed with palm of the hand in water holding position along with the thumb in opposition and touching the object held.

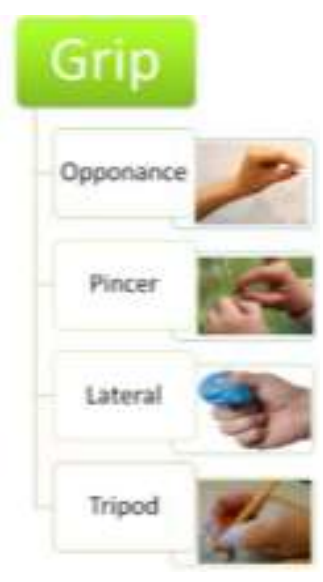

also called as three-jaw-chuck grip as it involves the tips of the three fingers.

\section{Occupational Therapy}

Occupational Therapy - A Part of Rehabilitation Team

A rehabilitation team working on a stroke patient comprises of physiatrists, physiotherapists, occupational therapists, prosthetist and orthoptist, speech therapist and many more, all having a different and complimentary role in the treatment. Out of these professionals first three i.e. physiatrist, physiotherapist and occupational therapist has the most important role in hand rehabilitation of a stroke patient and the occupational therapist plays the most conclusive role as he leads to patient towards the ultimate goal of rehabilitation that is establishment of the patient in his daily routine life amongst the family and the society.

An occupational therapist helps a patient to restore functional activities pertaining to activities of daily living including family, community, school, work, leisure and entertainment, etc. [University of Rochester Medical Center]. 


\begin{tabular}{|c|c|}
\hline \multicolumn{2}{|c|}{ Activities of Dally Living } \\
\hline Basic ADL & Instrumental ADL \\
\hline MOBILITY & HOME MANAGEMENT \\
\hline Bed Mobility & Shopping \\
\hline Wheelchair Mobility & Meal planning \\
\hline Transfers & Cleaning \\
\hline \multirow[t]{3}{*}{ Ambulation } & Laundry \\
\hline & Child care \\
\hline & Recycling \\
\hline SELF-CARE & COMMUNITY LIVING SKILLS \\
\hline Dressing & Money/financial management \\
\hline Self-feeding & Use of public transport \\
\hline Toileting & Driving \\
\hline Bathing & Shopping \\
\hline Grooming & Access to recreational activities \\
\hline COMMUNICATION & HEALTH MANAGEMENT \\
\hline Writing & Handling medication \\
\hline Typing & Knowing health risks \\
\hline Telephoning & Making medical appointments \\
\hline \multicolumn{2}{|l|}{ Use special communication devices } \\
\hline ENVIRONMENTAL HARDWARE & SAFETY MANAGEMENT \\
\hline Keys & Fire safety awareness \\
\hline Fancets & Ability to call emergency numbers \\
\hline Light switches & Response to fire calls \\
\hline Windows/doors & Identification of dangerous situations \\
\hline \multicolumn{2}{|l|}{ Refrigerator } \\
\hline & ENVIRONMENTAL HARDWARE \\
\hline & Vacuum cleaner \\
\hline & Can opener \\
\hline & Stove/oven operation \\
\hline
\end{tabular}

\section{Activities of Daily Living (ADL)}

ADL is referred to those activities which are performed routinely on daily basis or very frequently by all for e.g. activities related to personal hygiene, home care, job or work, studies, etc. These ADL are divided in two categories Basic Activities of Daily Living (B-ADL) and Instrumental Activities of Daily Living (I-ADL). The B-ADL includes those activities which does not need any technical knowledge like or least of it for e.g. dressing, operating light switches, telephoning, etc. On the other hand I-ADL are those which need some to a lot of skills and knowledge like shopping, child care, handling medicines, using stove/oven etc.

\section{Areas of Occupational Therapy Treatment}

A person has to perform some roles or some responsibilities in his life through which he establish himself in the family or society. But once he has encountered some disease especially any long lasting 
one like stroke, he find himself/herself lagging behind in their roles may be being an employee, a father/mother, a student, a son/daughter, a wife/husband or may any other role in his family or society. This kind of issues (especially stroke as a topic of this paper) grabs a person in physical, mental, psychological and financial agony which starts a vicious cycle of some unpleasant experiences which he had never encountered before.

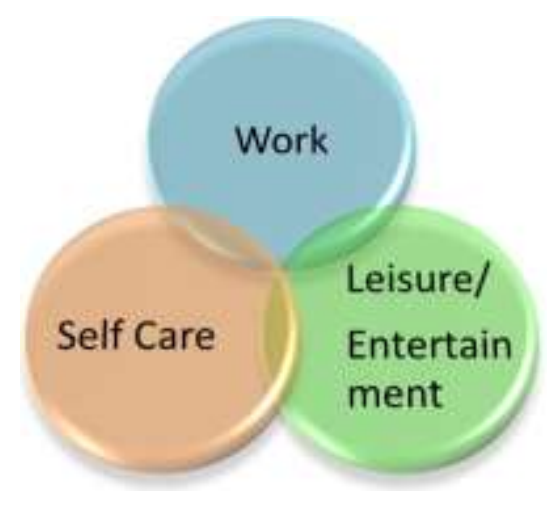

There are three main domains which are known as 'Areas of Occupational Therapy' viz. selfcare, work and leisure/entertainment in which every human being performs all the activities which are a part of three areas and work out to accomplish all the roles expected from them. Self-care includes the activities like bathing, brushing, clothing, toileting, etc. which a person perform to maintain himself for example person hygiene. Work include activities like earning money, studying, playing, home making, taking care of family members etc. e.g. a father may has to take care of the financial needs of his family, a mother has to take care of her children, a student has a work to study, play, enhance his physical and mental skills, etc.

Biomechanical Frame of Reference

Rehabilitation Frame of Reference

Neurodevelopmental Frame of Reference

Developmental Frame of Reference

Psychodynamic Frame of Reference

Behavioral Frame of Reference

Out of these, first two FORs viz. Biomechanical FOR and Rehabilitative FOR are used in the treatment of hand in stroke patients. Although here we have to take only fine motor skills thus only Biomechanical FOR will be discussed which is used in the evaluation and treatment of fine motor movements in stroke patients.

\section{Biomechanical FOR}

This FOR is used in the cases in which impairments in biomechanical body structures and functions that includes limited range of motion, decreased strength, structural instability and poor endurance lead to limitations in varies activities performed. This approach works on the basis that occupational performance of a person can be restablished by working on that limit activity performance [Hagedorn-1997, Trombly - 1995a]. For
Occupational Therapy professionals (called Occupational Therapists) work in three main areas i.e. through specialised techniques to treat underlying issues and to produce expected and sometimes miraculous results.

\section{Frames of Reference (FOR)}

Frames of reference act as guides to the Occupational therapists for evaluation, diagnosis and treatment of various types of conditions. Occupationally every patient is different and has different problems and expectations according to their past roles and present condition. There are total of eleven frames of reference used by occupational therapist namely:

\section{Cognitive Disability Frame of Reference Sensory Integration Frame of Reference Spatiotemporal Adaptive Frame of Reference Occupational Adaption Frame of Reference Model of Human Occupation}

an occupational therapist who is using this FOR to treat a stroke patient must have a good knowledge of the prognosis, pathology, precautions and about wound healing that can affect the treatment process. This helps in setting realistic and safe goals. Apart from that applied physical sciences that includes kinesiology and exercise physiology also helps to understand the biomechanics of the human body (Kielhofner - 1997).

Biomechanical FOR, on the treatment aspect includes adjunctive modalities like passive stretching which prepares the patient for occupational performance. Also enabling activities like peg boards, which are though not purposeful but practice of component skills needed for occupational performance can is done (Pedretti \& Early - 2001). Transitional activities help to ensure gains in 
biomechanical function during some spontaneous improvement in ADL.

To assess the amount of remediation of impairment which is required to reach the activity goals is determined by the use of activity analysis focusing on biomechanical aspects of the task (Pedretti - 2001). Treatment techniques like static orthotics prevent deformity by proper positioning of the joint and thus increase joint stability. Passive ROM can be increased by using dynamic orthotics (Coppard Lynn - 2001) and a variety of stretching exercises (Zemke -1995).
In stroke cases edema is another issues interferes with the smooth movements of the hand and fingers can be reduced through compressive wraps, elevation, active exercises and specialised massage. Activities eliciting the maximum contraction from the weak muscles improves strength of muscles. Stronger muscles should be made to work against resistance to enhance strength and the weak muscles must be assisted to help patient move (Kasch $\&$ Nickerson - 2001). Also activities which let the muscles work at the maximal contraction for longer time periods improve muscle endurance (Zemke 1995).

\begin{tabular}{|c|c|}
\hline \multicolumn{2}{|c|}{ Assessment Matrix } \\
\hline Component & Instrument / Tool \\
\hline \multirow[t]{3}{*}{ Muscle Strength } & Manual muscle testing \\
\hline & Grip strength \\
\hline & Pinch strength \\
\hline Range Of Motion (ROM) & Goniometer \\
\hline \multirow[t]{4}{*}{ Coordination } & Box \& Block Test \\
\hline & Nine hole peg test \\
\hline & Finger nose test \\
\hline & Rapid alternate movement test \\
\hline Tone & Modified Ashworth Scale \\
\hline Pain & Visual analogue scale \\
\hline \multirow[t]{3}{*}{ Sensation } & Monofilaments \\
\hline & Temperature tubes \\
\hline & Weighted boxes, etc \\
\hline \multirow[t]{2}{*}{ Edema } & Circumference \\
\hline & Volume \\
\hline \multirow[t]{2}{*}{ Motor Function } & Fugal Meyer assessment \\
\hline & Functional use of daily activities \\
\hline
\end{tabular}

\section{Evaluation and Assessment in Occupational Therapy}

Occupational therapists sets aims and objectives according to the condition in a particular disease. In the cases of stroke the objectives are:

- Evaluation of functional capacity - it is important to know the activities which were performed by the patient before the event of stroke and the residual capabilities after stroke. This includes muscle strength, joint range of motion, cutaneous sensations of hand, and even mental and psychological and financial conditions of the patient. This helps the therapist to set long term goals during the treatment process.

- Identification of areas \& role compromised - it helps to enlist and prioritise the components of the treatment process. It is based on survey methods and mainly subjective that includes family, friends, neighbors and even co-workers.

- Activities of Daily Living Evaluation Identification of activities performed in daily routine and then assessment of those activities 
through activity analysis methods helps to know the required constraints of certain activities and compare them with the residual capacities of the patient in order to design a treatment plan. For e.g. locking a door needs to grip the key and then turning it on the direction needed the patient needs an adequate lateral pinch strength and range of motion in the joints involved, proper tactile sensation, good coordination in the muscles of thumb and fingers, without any pain and other issues like edema. This kind of evaluation for every activity is done and with precise accuracy. [Role of occupational therapy after stroke Tennille J Rowland, Deirdre M Cooke, Louise A Gustafsson].

- Home Assessment - at the time of discharge from the hospital, the occupational therapist assesses the need of home assessment that includes the risk of falls, environmental barriers, impairments specific to the home environment and other needs of the patient and the care givers. This evaluation is needed to assess the capabilities of the patient and environmental constraints specific to the home environment. For e.g. patient's ability to plan and prepare meal will need consonance between the physical and mental status and the environment in the kitchen specifically. Similarly safe bed to toilet transfer and other similar activities are needed to the checked to set proper treatments later.

- Apart from the above gross assessments, other required assessments are also done which are listed in the table.

Techniques used in Occupational Therapy to improve fine motor skills in stroke patients

- Facilitates learning of skills - during the treatment process the patient is made to learn new activities and re-learn the activities which were performed by the patient before the condition. This method of learning is based on the neuroplasticity of the brain that states that "Neuroplasticity is the ability of the brain to undergo functional and structural changes in response to new experiences." [Klein JA, Jones TA]

- Training of Activities of Daily Living - after stroke it becomes very difficult for a patient to perform all the ADL due to many reasons like lack of muscle strength, altered sensation, imbalance of coordination, cognitive issues like memory and recall, etc. Training of ADL helps the patient to attain capabilities prior to event.

- Disability prevention - in most of the cases of stroke disabilities like limb deformities, pain, cognitive and perceptual disabilities, etc. occur due to decrease muscle power, spastic or flail muscles, hypo sensitivity of tactile sensations (like touch and temperature), etc. Proper and continuous occupational therapy intervention helps in prevention of these disabilities.

- Restore or reinforce functional capacity - as it is seen that the stroke survivors experience decreased performance in many areas occupational therapists help them to regain functional abilities and learn some new methods to perform activities in order to re-establish them in lost roles.

- Recommendation and training of Adaptive Devices - adaptive devices are objects or gadgets that compensate the disability after a stroke event. They are available in variety of range from simple reacher or utensil handles to complex devices like staircase lifts or motorised wheel chairs. Occupational therapist identifies the need and prescribe a proper adaptive device.

\section{Treatment Specifications}

The assessment matrix discussed above for evaluation contains some specific domains where occupational therapists focus. These domains are muscle strength, range of motion (ROM), coordination, tone, pain, sensation, edema, muscle endurance and motor functions. These domains are touched individually and in relation to each other as well. Specific treatment is prescribed and a plan is set including short term goals and long term goals. These goals are decided according to the previous activities done by the patient and the current condition of the hand and fingers. The main aim of these goals is to increase joint range of motion and muscle strength, improve sensation and coordination, enhance muscle endurance balance tone, and reduce pain and edema resulting in improved motor functions. Short term goals generally include picking up of small objects like beads, improve grips on pipes and handles, manipulate big and small objects in hand, etc. and long term goals will include operation of locks and keys, currency note counting, operate computers, prepare meal for the family, etc.

Although many activities can be designed to treat a particular issue but most of the activities can be used to address more than one issue. For example selection and sorting activity can be used to improve pinch, grasp, enhance muscle coordination, teach colours, shapes, improve cognition and perception, etc. To fulfill the treatment objectives of occupational therapy treatment for fine motor skills in stroke patients exercises and activities prescribed for their specific purpose are used. The following are some examples of the activities being used to address the corresponding issues 


\begin{tabular}{|c|c|}
\hline \multicolumn{2}{|c|}{ Treatment Specifications } \\
\hline Component & Treatment \\
\hline \multirow[t]{3}{*}{ Muscle Strength } & Passive stretching \\
\hline & Active mobilization \\
\hline & Force against resistance \\
\hline \multirow[t]{2}{*}{ Range Of Motion (ROM) } & Active mobilization \\
\hline & Maximal contraction \\
\hline \multirow[t]{2}{*}{ Coordination } & Enabling activities \\
\hline & Coordination specific exercises \\
\hline \multirow[t]{2}{*}{ Tone } & Active assisted movements \\
\hline & Active mobilization \\
\hline \multirow[t]{2}{*}{ Pain } & Active assisted movements \\
\hline & Compression garments \\
\hline Sensation & Sensory Integration \\
\hline \multirow[t]{4}{*}{ Edema } & Active mobilization \\
\hline & Compression garments \\
\hline & Limb elevation \\
\hline & Specialised massage \\
\hline \multirow[t]{2}{*}{ Endurance } & Maximal contraction \\
\hline & Force against resistance \\
\hline \multirow[t]{2}{*}{ Motor Function } & Enabling activities \\
\hline & ADL training \\
\hline
\end{tabular}

\section{CONCLUSION}

Stroke is one of the deadliest and most dangerous disease projecting numbers as third biggest cause of disability and second biggest cause of death throughout the world. It is a condition occurring due to sudden death of certain cells of brain due to lack of oxygen when the blood flow is lost or hindered due to blockage or rupture of the arteries of the brain. On the basis of causes of stroke there are two types of stroke: ischemic stroke and hemorrhagic stroke. Stroke leads to many physical, mental and psychological effects out of which loss of fine motor skills is the most disastrous consequence making the patient dependent on others. Fine motor skills is the ability to control movements of fingers and thumb through specialised movements through the coordinated function of nervous system and small muscles of hand. Alterations in fine motor skills can be caused due to loss of muscle strength, loss of muscle co-ordination, sensory alterations, cognitive disabilities, etc. leading to tremors uncontrolled movements of the wrist, fingers and the thumb thus patients are not able to perform the required activities as expected and to the maximum level of functionality.

Hand Functions are particularly those actions which lead to successful performance of different activities needed to achieve daily routine functions may be single handed or with both hands 
together. Hand functions are done to achieve four basic actions viz. reach, grip, manipulation/shift and release. The actions needed for the hand functions are divided into two main categories namely Grasp and Grip and further divided as spherical grasp, cylindrical grasp, hook grasp, lumbrical grasp and opponance grip, pincer/tip to tip grip and lateral grip.

A rehabilitation team working on a stroke patient comprises of physiatrists, physiotherapists, occupational therapists, prosthetist and orthoptist, speech therapist and many more, all having a different and complimentary role in the treatment. The occupational therapist plays the most conclusive role as he leads to patient towards the ultimate goal of rehabilitation that is establishment of the patient in his daily routine life amongst the family and the society.

Activities of Daily Living (ADL) is referred to those activities which are performed routinely on daily basis or very frequently for e.g. activities related to personal hygiene, home care, job or work, studies, etc. These ADL are divided in two categories Basic Activities of Daily Living (B-ADL) which includes activities like dressing, operating light switches, telephoning, etc. and Instrumental Activities of Daily Living (I-ADL) including activities like shopping, child care, handling medicines, etc.

There are three main domains to work for occupational therapists known as 'Areas of Occupational Therapy' viz. self-care, work and leisure/entertainment. Self-care includes the activities like bathing, brushing, clothing, toileting, etc. which a person perform to maintain himself for example person hygiene. Work include activities like earning money, studying, playing, home making, taking care of family members etc.

Frames of reference (FOR) act as guides to the Occupational therapists for evaluation, diagnosis and treatment of various types of conditions. There are total of eleven frames of reference used by occupational therapist out of which Biomechanical FOR is used in the treatment of fine motor movements in stroke patients. The evaluation of fine motor skills in a stroke patient in occupational therapy is done for functional capacity, areas and roles compromised, activities of daily living and home assessment. Also functional aspects of muscle strength, joint range of motion, coordination, tone, pain, sensation, edema and motor functions are also evaluated with the help of different instruments and tools.

The treatment process includes facilitation of learning skills, ADL training, disability prevention, restoration and reinforcement of functional capacities and recommendation and training of adaptive devices. The treatment specifications include passive stretching, active mobilization, enabling activities, sensory integration, compressive garments, specialised massage, ADL training, etc. As the treatment plans in occupational therapy is mostly activity based, the therapists use two types of activities, the purposeful like folding clothes, polishing shoes, donning on and off clothes, counting money, cleaning room, etc. and nonpurposeful activities like selection and sorting, beading, lacing, stacking, etc.

Occupational therapy as its specialty in hand rehabilitation has a wide range in its treatment protocol to pursue the treatment of a variety of areas of hand rehabilitation including fine motor skills after an event of stroke. The occupational therapist provides activity based treatment plans that help to regain motor function through a multidisciplinary approach focusing on training in a wide variety of aspects including muscle strength, range of motion, endurance, sensation, etc. to train the patient perform best in his activities of daily living. In turn the patient finds himself available to the family and society to work around in all the three occupational areas of self-care, work and leisure. Occupational therapy make use of different approaches called frames of reference for different types of treatments out of which biomechanical frame of reference is used as a base approach to improve fine motor skills. Occupational therapy plays a decisive role in the rehabilitation team to provide an opportunity to plan a proper treatment procedure for a stroke patient ultimately enhancing the restoration of fine motor skills with the help of a number of activities.

\section{REFERENCES}

1. In: Global Health Estimates; World Health Organization, 2012.

2. Jones, L.; Lederman, S. Human Hand Function; Oxford University Press: Oxford, 2007.

3. Kleim, J. A.; Jones, T. A. Principles of Experience-Dependent Neural Plasticity: Implications for Rehabilitation After Brain Damage. J. Speech Lang. Hear. Res. 2008, 51 (1).

https://www.jsmf.org/meetings/2008/may/Kleim\% 20\&\%20Jones\%202008.pdf, S225-S239.

4. Pedretti, L.; Early, M. Occupational Therapy, 5th ed; Mosby: St. Louis, 2001.

5. Rowland, T.; Cooke, D.; Gustafsson, L. Role of Occupational Therapy After Stroke. In Annals of Indian Academy of Neurology, 11th ed; Indian Academy of Neurology, Pp, 2008, pp 99-107.

6. Schell, B.; Gillen, G. Willard and Spackman's Occupational Therapy, 13th ed; Lippincott Williams \& Wilkins, 2018.

7. Semenko, B.; Thalman, L.; Ewert, E.; Delorme, R.; Hui, S.; Flett, H.; Lavoie, N., 2015. An Evidence Based Occupational Therapy Toolkit for Assessment and Treatment of the Upper Extremity Poststroke. Winnipeg Health Region Occupational Therapy Upper Extremity Working Group.

8. http://www.stroke.org Types of Stroke [Online]. https://www.stroke.org/en/about-stroke/types-ofstroke (accessed Dec 29 2020), 2020. 
9. Thai, J. N.; Pacheco, J. A.; Margolis, D. S.; Swartz, T.; Massey, B. Z.; Guisto, J. A.; Smith, J. L.; Sheppard, J. E. Evidence-Based Comprehensive Approach to Forearm Arterial Laceration. West. J. Emerg. Med. 2015, 16 (7), $1127-1134$. 